\title{
Growth hormone: a newly identified developmental organizer
}

\author{
Rajat K Das*, Sarmistha Banerjee* and Bernard H Shapiro \\ Department of Biomedical Sciences, University of Pennsylvania, School of Veterinary Medicine, \\ Philadelphia, Pennsylvania, USA \\ *(R K Das and S Banerjee contributed equally to this work)
}

Correspondence should be addressed to B H Shapiro

Email

shapirob@vet.upenn.edu

\begin{abstract}
The sexually dimorphic expression of cytochromes P450 (CYP) drug-metabolizing enzymes has been reported in all species examined. These sex differences are only expressed during adulthood and are solely regulated by sex differences in circulating growth hormone (GH) profiles. Once established, however, the different male- and female-dependent CYP isoform profiles are permanent and immutable, suggesting that adult CYP expression requires imprinting. As the hormone that regulates an adult function is likely the same hormone that imprints the function, we selectively blocked GH secretion in some newborn male rats, whereas others received concurrent physiologic replacement of rat $\mathrm{GH}$. The results demonstrate that adult male $\mathrm{GH}$ activation of the signal transduction pathway regulating expression of the principal CYP2C11 isoform is obligatorily dependent on perinatal GH imprinting, without which CYP2C11 and drug metabolism would be permanently and profoundly suppressed. As there are other adult metabolic functions also regulated by GH, pediatric drug therapy known to disrupt GH secretion could unintentionally impair adult health.
\end{abstract}

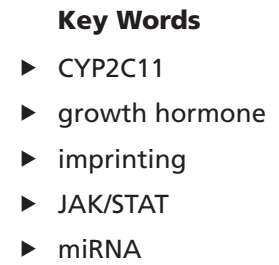

Journal of Endocrinology (2017) 232, 377-389

\section{Introduction}

Hormonal imprinting refers to a biological process in which the target tissue becomes responsive to the hormone. During the initial exposure, the hormone irreversibly reprograms the development of the affected tissue so as to permanently alter some functional aspect normally responsive to the hormone and often establishing a sexual dimorphism (Csaba 2008). Moreover, the tissue is programmable for only a brief developmental period, after which time the tissue becomes permanently unresponsive to imprinting (Goldman 1970, Ohno 1979, Feder 1981, Shapiro 1985a). Imprinting alone, however, is generally insufficient to ensure the expression of the programmed function. Accordingly, expression of the affected function requires both imprinting and activation, the latter being a reversible process, but required to express the imprinted function. For example, perinatal testosterone or one of its metabolites is required to permanently 'wire' or imprint the male brain to exclusively express masculine sexual behavior. However, the brain has to be stimulated or activated in adulthood by the same hormone to elicit the imprinted male sexual behavior. Both imprinting and activation are required for normal masculine sexual behavior (Shapiro et al. 1980, Feder 1981, Shapiro 1985a), which explains why perinatally castrated males and intact females, not hormonally imprinted, are unable, regardless of adult hormone treatment, to exhibit normal masculine sexual behavior (Shapiro et al. 1980, Feder 1981).

The cytochromes P450 (CYP) monooxygenases are an ancient family of heme enzymes that catalyze a large variety of essential metabolites, which include the synthesis and the deactivation of adrenal, gonadal and thyroid hormones, eicosanoids, bile acids and fatty acids, as well as the detoxification of innumerable drugs and environmental chemicals; hence, their ubiquitous

Published by Bioscientifica Ltd 
presence throughout all species, where hundreds of isoforms have evolved to catalyze a countless number of substrates (Nebert \& Dieter 2000, Hannemann et al. 2007). In the case of mammals, including humans, the CYPs are most prominent in the liver, where they are expressed during adulthood as dozens of different isoforms, many of which are sexually dimorphic (sex differences in CYP expression occurs postpubertally) (Shapiro et al. 1995). The only endogenous factor known to regulate the expression of adult hepatic CYP is growth hormone (GH) (Legraverend et al. 1992, Shapiro et al. 1995). At puberty, males begin to secrete GH in what is referred to as an 'episodic' profile characterized by several daily bursts of hormone separated by lengthy undetectable or barely detectable GH concentrations. In contrast, the adult female GH profile is considered 'continuous,' as there are far more secretory bursts, often at lower amplitudes, of the hormone separated by briefer interpulse periods, often containing measurable levels of GH. In fact, these sex differences in the circulating GH profile are solely responsible for the sex difference in CYP expression and resulting drug metabolism observed during adulthood (Legraverend et al. 1992, Shapiro et al. 1995). That is, the masculine 'episodic' $\mathrm{GH}$ profile induces the expression of the male-specific CYP isoforms, whereas the feminine 'continuous' GH profile induces the expression of the female-specific CYP isoforms. In this regard, we previously reported that adult male rats and men cannot be induced (regardless of GH treatment) to express the normal female profile of hepatic CYPs (Pampori \& Shapiro 1999, Thangavel et al. 2004, 2011, Dhir et al. 2006, Thangavel \& Shapiro 2008) nor can adult female rats or women be induced to fully express the masculine CYP profile (Dhir et al. 2006, 2007, Thangavel et al. 2006, Thangavel \& Shapiro 2007). The response of each of the dozen or so sex-dependent rat CYP isoforms to $\mathrm{GH}$ regulation has been described (Thangavel \& Shapiro 2008, Das et al. 2013a, Banerjee et al. 2015). If CYP enzymes were not imprinted, then irrespective of sex, the same adult treatment should produce the same CYP expression levels in males and females. As this is not the case, we have concluded that the sex differences in adult expression profiles of CYPs are permanent and irreversible, a condition demonstrating imprinting. Because an adult tissue/function is apt to be responsive to the same hormone by which it was imprinted, we have proposed, for the first time, that like androgens, perinatal GH is a developmental organizer required for the normal expression of adult CYP-dependent drug metabolism.

\section{Materials and methods}

\section{Animal experimentation}

Rats were housed in the University of Pennsylvania Laboratory Animal Resources facility under the supervision of certified laboratory animal medicine veterinarians and were treated according to a research protocol approved by the Institutional Animal Care and Use Committee of the University. Housing conditions, as well as breeding and treatment protocols, were reported previously (Shapiro et al. 1989, Pampori et al. 1991b). Basically, neonatal male rat pups were injected, sc, with either of 2 of 4 possible treatments. (1) Monosodium glutamate (MSG), $4 \mathrm{mg} / \mathrm{g} \mathrm{bwt}$ on alternate days, starting within $24 \mathrm{~h}$ of birth for a total of 5 injections. (2) An equivalent amount of the MSG NaCl diluent. (3) Rat growth hormone $(\mathrm{GH}), 20 \mu \mathrm{g} / 100 \mathrm{gbwt}$, twice/day for the first 12 days of life. (4) An equivalent amount of the GH bicarbonate diluent. Accordingly, control pups received treatments 2 and 4; MSG pups received treatments 1 and 4; GH pups received treatments 2 and 3 and MSG + GH pups received treatments 1 and 3 . Body weights, Lee indices and hexobarbital sleep times were determined as previously reported (Shapiro et al. 1989, Pampori et al. 1991b).

\section{Growth hormone determinations}

Plasma GH concentrations were determined by a sensitive sandwich ELISA method as described previously (Das et al. 2013a). Procedures for collecting unstressed postnatal plasma as well as adult serial blood sampling for GH determinations have been reported (Pampori et al. 1991a, Banerjee et al. 2015).

\section{Primary hepatocyte cultures}

Preparation of hepatocytes from 18-week-old male rats was performed by in situ perfusion of collagenase

Table 1 Source of antibodies.

\begin{tabular}{|c|c|}
\hline Name of antibodies & Source \\
\hline CYP2C11 & Detroit R\&D Inc., Franklin, MI, USA \\
\hline STAT5b & Santa Cruz Biotechnology \\
\hline Anti-phosphotyrosine & EMD Millipore \\
\hline CIS & Santa Cruz Biotechnology \\
\hline SOCS2 & Santa Cruz Biotechnology \\
\hline$\beta$-Actin & Sigma Chemical \\
\hline
\end{tabular}

Published by Bioscientifica Ltd. 
Table 2 Primer sequences used for qRT-PCR.

\begin{tabular}{lll}
\hline mRNA & Forward $\left(5^{\prime},>3^{\prime}\right)$ \\
\cline { 1 - 2 } Cis & AGCCATGCAGCCCTTAC \\
Socs2 & TTAAAAGAGGCGCCAGAAGGA \\
Socs3 & CCTCCAGCATCTTTGTCGGAAGAC \\
Cyclophilin A & CGACTGTGGACAGCTCTAAT
\end{tabular}

Reverse $\left(5^{\prime}>>3^{\prime}\right)$

CTAGCACCTTCGGTTCATTCT GCCCGGCTGATGTCTTAACA TACTGGTCCAGGAACTCCCGAATG CCTGAGCTACAGAAGGAATG through the portal vein of anesthetized rats. The viability of the initial cell suspension of hepatocytes was typically between $80 \%$ and $90 \%$ with trypan blue. The hepatocytes were plated at a density of $3 \times 10^{6}$ viable cells per well in 6-well plates previously coated with Matrigel. Medium, cell isolation and culture conditions were reported previously (Thangavel et al. 2006, Thangavel \& Shapiro 2007).

\section{Hormonal conditions}

To replicate the masculine episodic GH profile, hepatocytes were exposed to recombinant rat $\mathrm{GH}$ (0.2 ng/mL; National Hormone and Peptide Program, Torrance, CA, USA) for $30 \mathrm{~min}$, followed by 2 careful washings with $\mathrm{GH}$-free medium that remained in the wells for $11.5 \mathrm{~h}$, at which time the cells were again washed and exposed to the next $30 \mathrm{~min}$ pulse of $\mathrm{GH}$. On the 6 th day, cells were harvested $30 \mathrm{~min}$ after the addition of the last GH pulse. Following the same treatment schedule, control cells were exposed to the GH vehicle alone (Thangavel et al. 2006, Thangavel \& Shapiro 2007).

\section{Western blot and immunoprecipitation}

Whole-cell lysates and nuclear fractions (Thangavel \& Shapiro 2007, Banerjee et al. 2015) were extracted from freshly isolated preplated hepatocytes as well as cultured primary hepatocytes $30 \mathrm{~min}$ after the last $\mathrm{GH}$ pulse. Whole-cell lysate protein was electrophoresed and electroblotted onto nitrocellulose membranes for immunoblotting. Accordingly, the blots were probed with antibodies against CYP2C11 and anti-suppressors of cytokine signaling (CIS and SOCS2). Nuclear fractions were immune precipitated with signal transducers and activators of transcription 5b (STAT5b) antibodies as described previously (Thangavel \& Shapiro 2007, Banerjee et al. 2015). Next, the immunoprecipitates were probed with anti-phosphotyrosine. The protein signals were scanned and the densitometric units were obtained as integrated density values. Antibodies and their vendors are listed in Table 1.

\section{Quantitative real-time PCR (qRT-PCR)}

Cyp2c11, Cis, Socs1, Socs2, Socs3 and Cyclophilin A (housekeeping gene) expression were determined by qRT-PCR according to our previously report procedures (Das et al. 2013b, Banerjee et al. 2015). The primer sequences used to measure gene expression are listed in Table 2 or reported previously (Waxman \& Frank 2000, Das et al. 2013a).

\section{Chromatin immunoprecipitation (ChIP)}

A ChIP assay was performed to determine the intensity of STAT5b binding to the Cyp2c11 promoter on cultured hepatocytes $30 \mathrm{~min}$ after the last episodic $\mathrm{GH}$ pulse on the 6th day in culture as previously described (Thangavel \& Shapiro 2007, Banerjee et al. 2015).

\section{MicroRNA (miRNA)}

Due to their possible association with CYP2C11 and GH expression and/or action, we measured the expression levels of miRNA-141-3p, miRNA-130a-3p, miRNA-381-3p and miRNA-29b-3p in freshly isolated hepatocytes as well as cultured hepatocytes exposed to the masculine-like $\mathrm{GH}$ profile according to our previous methods (Banerjee et al. 2015). The primers for the miRNAs and procedural controls are presented in Table 3.

\section{Statistics}

All data were subjected to ANOVA. Significant differences were determined with $t$ statistics and the Bonferroni procedure for multiple comparisons.

Table 3 Source of miRNA primersa.

\begin{tabular}{l} 
miRNA \\
\hline miR-141-3p \\
miR-130a-3p \\
miR-381-3p \\
miR-29b-3p \\
Reverse transcriptase \\
control \\
PCR control
\end{tabular}

Source
Rn_miR-141_1 miScript primer assay
Rn_miR-130a_1 miScript primer assay
Rn_miR-381_2 primer assay
Rn_miR-29b_1 miScript primer assay
Ctrl_miRTC_1 miScript primer assay
Hs_SNORD96A_11 miScript primer assay

aFrom Qiagen Sciences, Frederick, MD, USA.

Published by Bioscientifica Ltd 


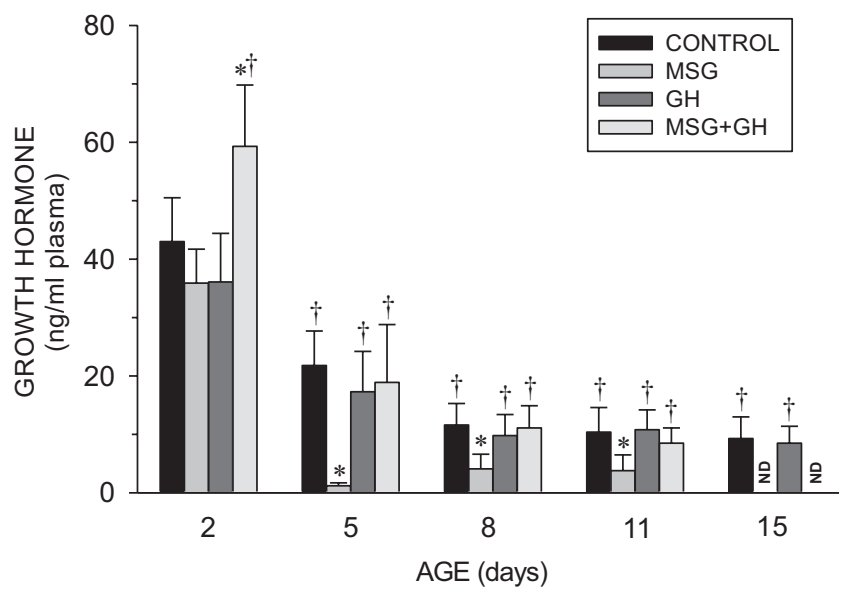

Figure 1

Plasma growth hormone $(\mathrm{GH})$ of male rat pups treated with diluents (CONTROL), MSG and/or GH. Neonatally treated pups were injected with either MSG ( $4 \mathrm{mg} / \mathrm{g} \mathrm{bwt}$ ) or an equivalent dose of diluent on postnatal days 1, 3, 5, 7 and 9; rat GH ( $20 \mu \mathrm{g} / 100 \mathrm{~g} \mathrm{bwt})$ and/or the $\mathrm{GH}$ diluent, twice/d for the first 12 days of life. Results are presented as the means \pm S.D. of at least 7 pups/group. ${ }^{*} P<0.01$ vs CONTROL; ${ }^{+} P<0.01$ vs MSG treatment alone.

\section{Results}

\section{Immediate and long-term suppression of GH secretion by neonatal MSG}

In agreement with previous (Oliver et al. 1982, Kacsoh et al. 1989) as well as our own report (Banerjee et al. 2015), plasma GH levels in control groups were maximum the first few postpartum days of life, thereafter declining $\sim 50 \%$ by 5 days of age to $75-80 \%$ from 8 through 15 days of age (Fig. 1). GH levels of the MSG-treated neonates at
2 days of age, which was often less than $24 \mathrm{~h}$ after the first MSG injection, were normal. Thereafter, circulating GH concentrations declined 90-95\% (postnatal day 2 through 11 days of age), to undetectable (15 days of age). In spite of the administration of exogenous GH during the first 12 days of life, circulating levels of the hormone were the same as controls. This seemingly unexpected finding could be explained by the presence of a functional hypothalamic-pituitary GH feedback loop in which the administered GH suppressed the secretion of endogenous hormone (Oliver et al. 1982, Wehrenberg \& Giustina 1992) and/or by the fact that blood collections occurred $12 \mathrm{~h}$ after the last $\mathrm{GH}$ injection at which time the exogenous hormone could have cleared from the circulation (Brownborg et al. 1993). Concomitant administration of MSG and GH resulted in essentially normal-like levels of circulating hormone until the GH was withdrawn, and like the MSG treatment alone, plasma GH concentrations were now undetectable in the pups. (The $\sim 30 \%$ abovecontrol levels of plasma GH in the 2-day-old pups treated with MSG + GH could be due to a combination of the injected hormone and any transient residual GH from the MSG treatment).

Adult plasma GH profiles are presented as schematic representations of the actual circulating profiles (Fig. 2). The control rats secreted the typical adult masculine profile (Jansson et al. 1985a, Agrawal et al. 1995, Shapiro et al. 1995) characterized by episodic pulses containing amplitudes of $\sim 200 \mathrm{ng} / \mathrm{mL}$ with durations of $\sim 1 \mathrm{~h}$, separated by interpulse periods of $2-3 \mathrm{~h}$ devoid of measurable hormone. In contrast, none of the adult neonatally MSG-treated rats had any measurable plasma
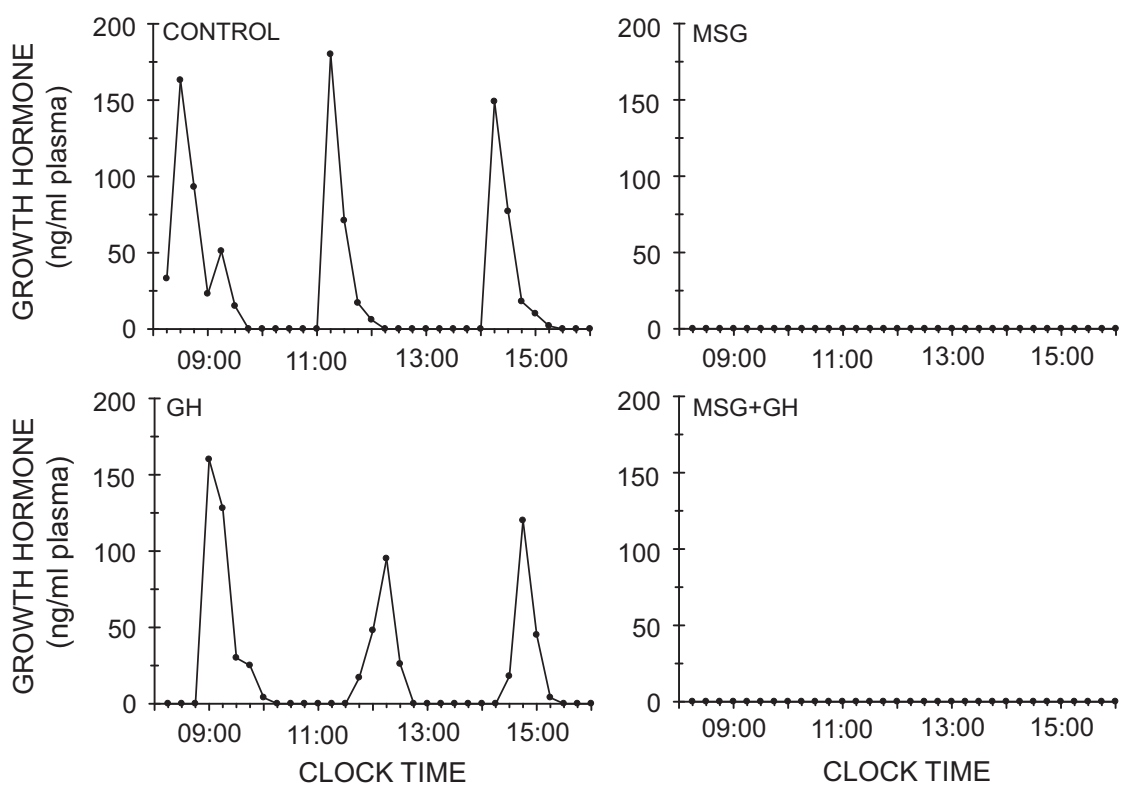

Figure 2

Adult plasma growth hormone $(\mathrm{GH})$ profiles in growth hormone-depleted neonates. Plasma levels of circulating $\mathrm{GH}$ were obtained from individual, undisturbed catheterized adult rats neonatally treated with diluents (CONTROL), MSG and/or GH for 8 continuous hours at 15-min intervals. Similar results were obtained from 4 to 5 additional animals in each treatment group. Figure 1 for details of neonatal treatment. 
GH $(>0.125 \mathrm{ng} / \mathrm{mL})$ during the 8 continuous hours of serial sampling. Adult rats, administered GH for the first 12 days of life, secreted the typical masculine episodic GH profile with the exception of pulse amplitudes $\sim 30 \%$ $(P<0.01)$ lower than controls. The combined neonatal treatment of MSG and GH resulted in adult GH profiles identical to MSG alone, i.e., no detectable plasma GH.

\section{Neonatal GH mitigates neonatal MSG-induced adult obesity}

The Lee index is a measure of obesity; the higher the value, the greater the obesity. At all ages measured
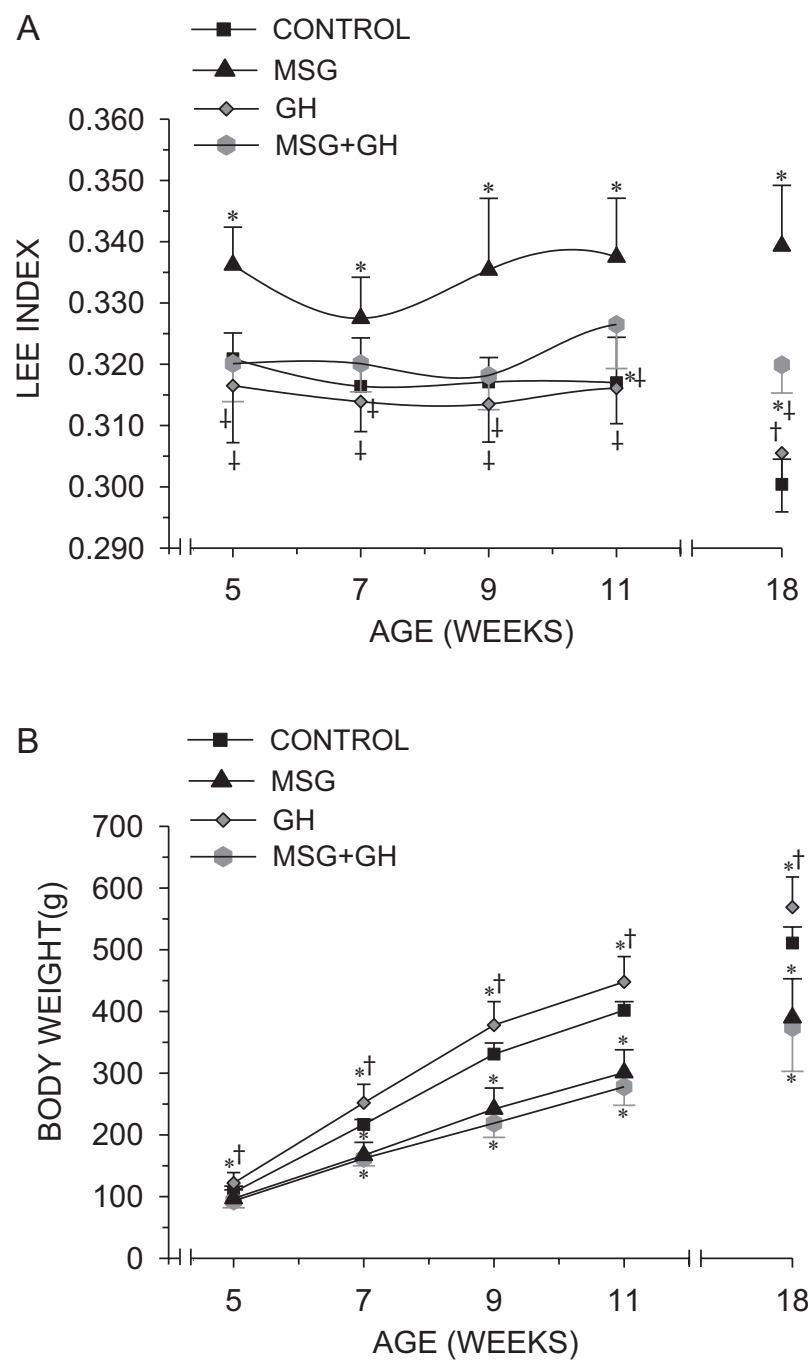

Figure 3

Growth hormone $(\mathrm{GH})$ imprinting affects post-weaning maturational Lee index profiles (A) and body weight gains (B). Results are presented as the means \pm S.D. of at least 15 rats/group. CONTROL, MSG and/or GH treatments are described in detail in Fig. $1 .{ }^{*} P<0.01$ vs CONTROL; ${ }^{+} P<0.01$ vs MSG treatment alone.
(5-18 weeks of age), the Lee index of the neonatally MSG-treated rats was significantly $(P<0.01)$ greater than age-matched controls (Fig. 3A); this is not likely a result of overeating (Olney 1969), but rather a consequence of thermoregulation of a lower than normal body temperature and a general lethargy (Tokuyama \& HimmsHagen 1986) preventing normal body weight gain. The Lee indices of the GH-alone-treated rats were no different from controls. In spite of the absence of any detectable plasma GH, adult rats neonatally exposed to both MSG and GH exhibited no measurable obesity at 5, 7 and 9 weeks of age; thereafter (11 and 18 weeks of age), their Lee indices were intermediate $(P<0.01)$ between controls and MSG alone, suggesting that the hormone imprints the 'obesity set point' attenuating the effects of both neonatal and adult GH depletion observed in MSG-alonetreated rats.

In contrast to its effect on obesity, neonatal GH was unable to moderate the inhibitory action of MSG on body weight gain, i.e. an index of general (e.g. musculoskeletal) body growth (Fig. 3B). Consequently, adult body weight gain of male rats neonatally treated with either MSG or both MSG and GH were no different, but significantly $(P<0.01$ at all ages) less than controls which in turn were less than the GH alone-treated rats, the latter treatment producing the greatest body weight gain.

\section{Neonatal GH imprints adult responsiveness of hepatic CYP2C11 to episodic $\mathrm{GH}$ regulation}

Hexobarbital-induced sleep time is an in vivo measure of drug metabolism competence. When given the same dose of hexobarbital per $\mathrm{kg}$ of $\mathrm{b}$ wt, adult rats neonatally treated with MSG slept 3 times as long as control rats (Fig. 4A1), indicating a considerable deficiency in CYP isoforms, particularly CYP2C11 (Ryan \& Levin 1990, Pampori et al. 1991b), which greatly contributes to hexobarbital metabolism, i.e., hydroxylation. Neonatal treatment of MSG and GH resulted in adult sleeping times that were about twice as long as controls but still significantly briefer than the MSG alone treatment, indicating the expression of some level of hexobarbital hydroxylase capacity, e.g., CYP2C11.

CYP2C11 is the principal male-dependent form, comprising $>50 \%$ of the total CYPs in adult male rat liver (Morgan et al. 1985), but undetectable in female liver, explaining its designation as male specific (Legraverend et al. 1992). (CYP2C11 is expressed only in adulthood and its full expression is solely dependent on the circulating masculine GH profile whose secretion

Published by Bioscientifica Ltd. 
A1

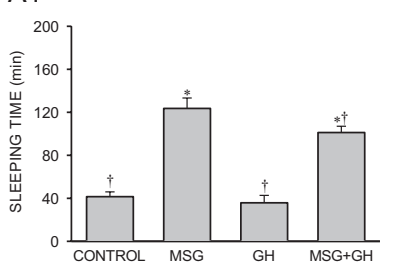

B1

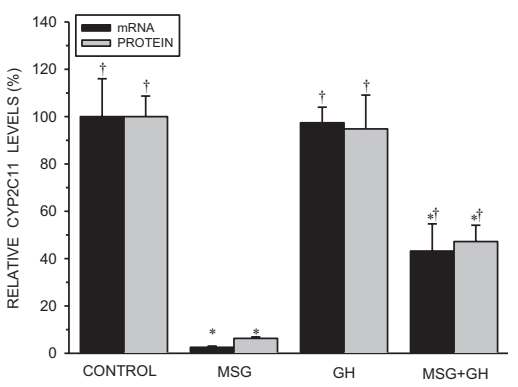

B2

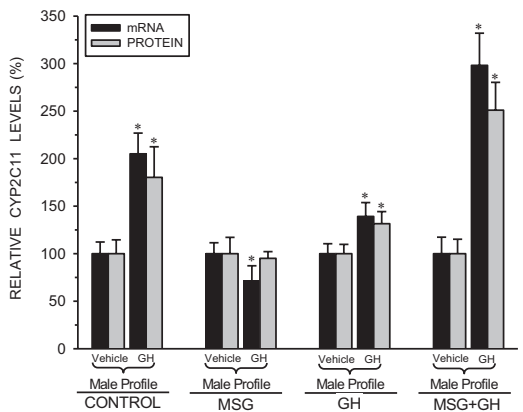

Figure 4

Neonatal growth hormone (GH) imprints adult hepatic CYP2C11 expression. (A1) CYP2C11-dependent hexobarbital-induced sleeping times in adult rats neonatally administered diluents (CONTROL), MSG and/or GH determined following an i.p. injection of hexobarbital (150 mg/kg b wt). Results are presented as the means \pm S.D. of at least 10 rats/group. ${ }^{*} P<0.01$ vs CONTROL; ${ }^{+} P<0.01$ vs MSG treatment alone. (B1) Relative expression levels (mRNA and protein) of the paramount male-specific CYP2C11 isoform in freshly isolated preplated hepatocytes from adult male rats neonatally administered diluent (CONTROL), MSG and/or GH. Results are presented as a percentage of mRNA or protein in hepatocytes from CONTROL rats arbitrarily designated as $100 \%$. Values are means \pm S.D. of at least 6 rats/data point. ${ }^{*} P<0.01$ vs CONTROL; ${ }^{+} P<0.01$ vs MSG treatment alone. (B2) Hepatocytes from the same rats as (B1) above were exposed to either the episodic male-like GH profile or the hormone's vehicle for 6 days in culture. Results are presented as a percentage of mRNA or protein in hepatocytes exposed to vehicle arbitrarily designated $100 \%$. Values are means \pm s.D. of at least 6 rats/data point. ${ }^{*} P<0.01$ vs hepatocytes exposed to the episodic vehicle profile from rats of the same neonatal treatment, i.e. CONTROL, MSG, GH or MSG + GH as described in Fig. 1.

occurs only postpubertally (Legraverend et al. 1992, Shapiro et al. 1995)).

As freshly isolated hepatocytes, CYP levels in vitro reflect in vivo concentrations (Thangavel et al. 2006). Accordingly, expression of episodic GH-dependent CYP2C11 was profoundly reduced in freshly isolated hepatocytes from GH-devoid, neonatally MSG-treated adult male rats (Fig. 4B1). In contrast, neonatal administration of both MSG and GH resulted in substantial expression levels of CYP2C11 ( 40\% of control values) in spite of the same total absence of circulating GH as observed in the MSG-treated rats. In this regard, a minimal baseline level of the hepatic CYP2C11 ( 20-40\% of normal) can be expressed in the absence of $\mathrm{GH}$, whereas full induction of the isoform requires exposure to the masculine episodic GH profile (Waxman et al. 1991, Agrawal \& Shapiro 2000). Consequently, these findings indicate that neonatal $\mathrm{GH}$ programs developmental mechanisms responsible for expression of GH-independent, baseline CYP2C11 expression. Neonatal GH treatment alone, however, had no effect on in vivo-like adult expression of CYP2C11.

After 6 days of exposure to the male-like episodic GH profile, CYP2C11 expression levels in the primary heptaocytes derived from control rats doubled above vehicle-treated baseline concentrations (Fig. 4B2). The inability of the masculine GH profile to induce CYP2C11 expression in hepatocytes from MSG-alone-treated males indicates an irreversible repression of the enzyme. In contrast, hepatocytes derived from adult males neonatally treated with both MSG and GH responded to the episodic $\mathrm{GH}$ profile with a $\geq 2.5$-fold increase in CYP2C11 expression. The only difference between the MSG-alone group and the MSG plus GH group was the presence in the latter of $\mathrm{GH}$ during the critical postnatal developmental period, demonstrating the imprinting action of postnatal $\mathrm{GH}$ on adult function. Recall, both groups were lacking GH during their immature, peripubertal and adult lives. (The difference between CYP2C11 induction levels in the control and the combined MSG and GH treatment group is not as dramatic as it appears. Induction levels are based on vehicle exposure levels designated 100\% in all groups. In fact, vehicle treatment of levels of CYP2C11 in the combined MSG and GH group were considerably lower $(\sim 35 \%)$ than that in the control group resulting in a calculated higher GH induction level in the combined treatment group).

\section{Neonatal GH irreversibly imprints the STAT5b signal transduction pathway mediating episodic $\mathrm{GH}$ induction of CYP2C11}

The terminal transducer in the signaling transduction pathway mediating episodic GH induction of CYP2C11 is STAT5b. This transcription factor must be activated (i.e., phosphorylated), homodimerized and translocated to the nucleus, where it binds to a promoter site initiating the transcription of the Cyp2c11 gene (Waxman \& Frank 2000). Primary hepatocytes were cultured in the presence 

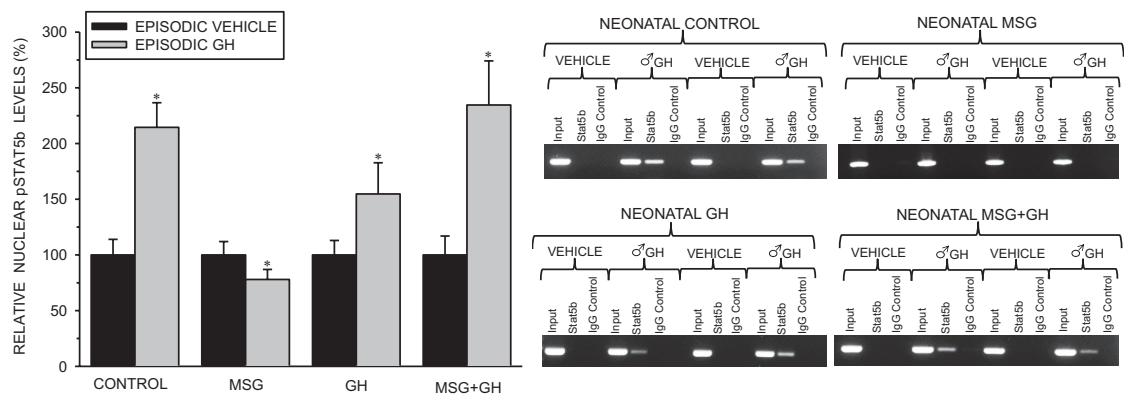

\section{Figure 5}

Neonatal growth hormone (GH) imprints adult growth hormone regulation of STAT5b activation, nuclear translocation and binding to the CYP2C11 promoter. (Left panel) Nuclear levels of activated STAT5b (pSTAT5b) in cultured primary hepatocytes exposed to either the episodic male-like GH profile or the episodic profile containing GH vehicle alone for 6 days. Results are presented as a percentage of nuclear pSTAT5b in hepatocytes exposed to vehicle arbitrarily designated as $100 \%$. Values are means \pm s.D. of at least 6 rats/data point. ${ }^{*} P<0.01$ vs hepatocytes exposed to the episodic vehicle GH profile from rats of the same neonatal treatment, i.e. CONTROL, MSG, GH or MSG + GH as described in Fig. 1. (Right panel) Representative ChIP blots (2 of 5/group) demonstrating episodic GH regulation ( ${ }^{\star} \mathrm{GH}$ ) described above, of pSTAT5b binding to the Cyp2c11 promoter in hepatocytes derived from adult male rats neonatally treated with either diluents (CONTROL), MSG, GH or MSG + GH as described in Fig. 1.

of the masculine episodic GH profile or episodic GH vehicle alone for 6 days. The cells were harvested $30 \mathrm{~min}$ after the last GH pulse; an optimal time to measure the STAT5b response (Verma et al. 2005). In agreement with the transduction requirements of GH-induced transcription of CYP2C11, the concentration of activated nuclear phospho-STAT5b (pSTAT5b) increased 2-fold in episodic GH-exposed hepatocytes from control rats, whereas the same hormone treatment produced a $20-25 \%$ decline in nuclear levels of pSTAT5b in adult hepatocytes from neonatal MSG-alone-treated rats (Fig. 5). In contrast, exposure of adult hepatocytes from the combined neonatal MSG and GH treatment to episodic GH resulted in a 2-fold increase in nuclear pSTAT5b concentrations, similar to control hepatocytes. Neonatal GH treatment alone, however, resulted in only $\sim 50 \%$ increase in the nuclear translocation of pSTAT5b reflecting the lower induced levels of CYP2C11 (Fig. 4B2).

The binding of pSTAT5b to the Cyp2c11 promoter was evaluated in adult hepatocytes from the various neonatal treatments by ChIP assays (Fig. 5, right). In agreement with the expected sequence of signaling events, robust binding of pSTAT5b to the promoter occurred in control hepatocytes exposed to the masculine-like GH profile. In contrast, none of the episodic GH-treated hepatocytes from the MSG-treated rats exhibited detectable binding of the activated transcription factor to the Cyp2c11 promoter. Hepatocytes from the combined neonatal treatment $(\mathrm{MSG}+\mathrm{GH})$ were found to display pSTAT5b promoter-binding levels similar to those of hepatocytes from control male rats. Lastly, the density of the pSTAT5b bands in the ChIP assays in hepatocytes from the $\mathrm{GH}$-alone-treated rats was somewhat lower than that of bands from control and combined MSG- and GH-treated rats, indicating reduced binding, in agreement with the hepatocytes' lower levels of both nuclear pSTAT5b and CYP2C11 expression.

\section{Developmental GH irreversibly imprints expression of the SOCS family of inhibitory proteins mediating GH action}

The SOCS family of inhibitory proteins are negative regulators of GH signaling. Their actions are particularly important in regulating the effects of episodic GH on such male-dependent parameters as growth, musculoskeletal metabolism and CYP expression (Karlsson et al. 1999, Waxman \& Frank 2000). Consequently, we decided to examine the long-term effects of neonatal GH on both the in vivo-like levels and in vitro responsiveness of four of the most commonly referenced SOCS, i.e. CIS, SOCS1, SOCS2 and SOCS3 (Fig. 6). Neonatal GH ablation (i.e. MSG treatment) had little effect on both the in vivo and in vitro adult expression of hepatic SOCS3 (Fig. 6D1 and D2), which may explain our earlier report demonstrating the ineffectiveness of the masculine episodic GH profile to regulate SOCS3 expression (Thangavel \& Shapiro 2007). In contrast, the absence of circulating $\mathrm{GH}$ in adulthood of both the MSG and MSG + GH neonatal treatment groups resulted in lower CIS, SOCS2 and SOCS1 in vivo expression levels, most dramatically at the transcription level (Fig. 6A1, B1 and C1), a possible result of altered ubiquitination levels shown to be regulated by $\mathrm{GH}$ (Banerjee et al. 2013). These SOCS proteins are known to be induced by the episodic GH profile, but only in males and not in females even when females are renaturalized

Published by Bioscientifica Ltd. 

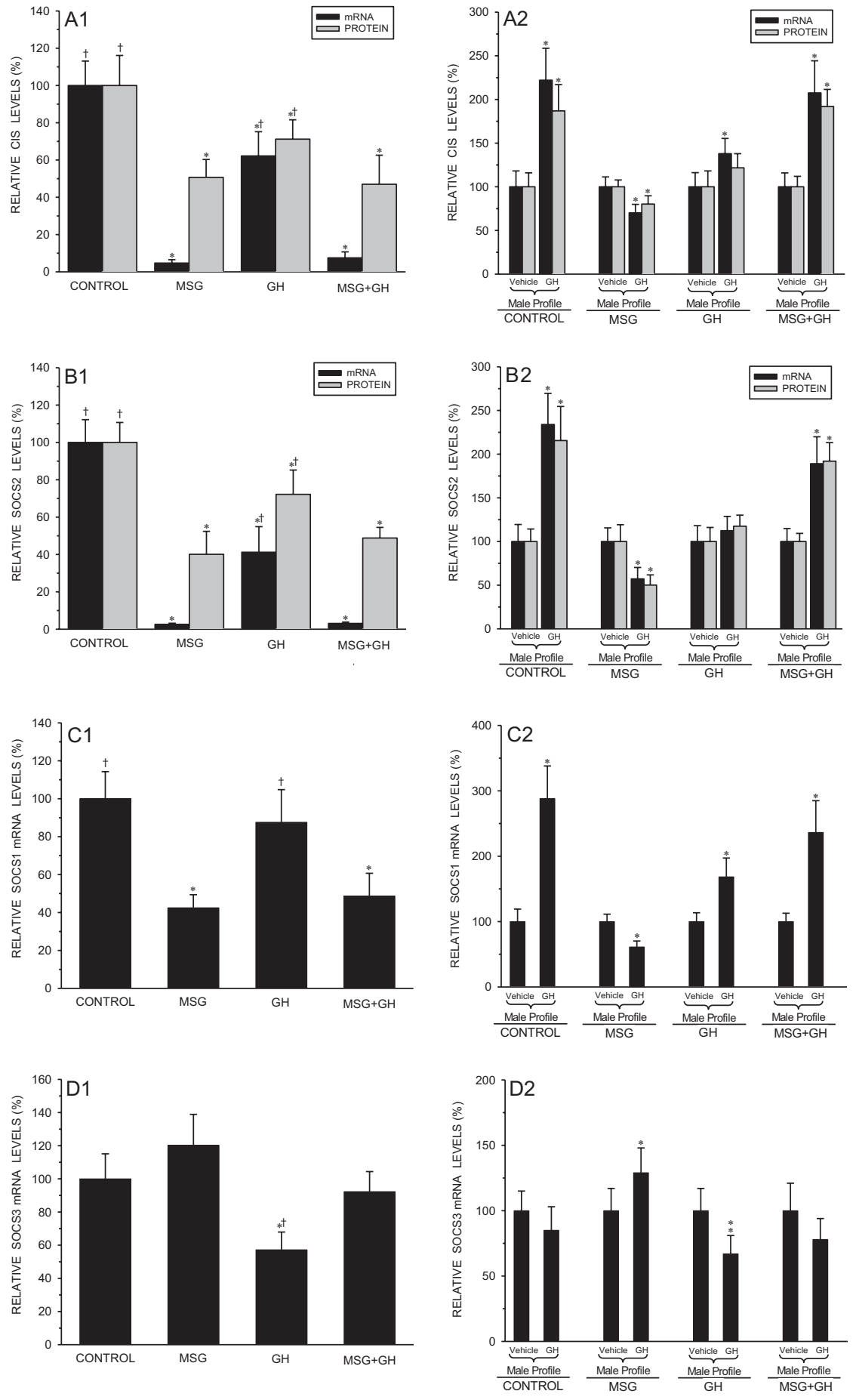

Figure 6

Neonatal growth hormone (GH) imprints adult growth hormone regulation of CIS/SOCSs expression. (A1, B1, C1, D1) Levels of 4 members of the CIS/SOCS family (i.e. CIS, SOCS2, SOCS1 and SOCS3) were measured in freshly isolated preplated hepatocytes from adult male rats neonatally administered diluents (CONTROL), MSG, GH or MSG + GH as described and statistically analyzed in Fig. 1. (A2, B2, C2, D2) Concentrations of the same CIS/SOCS family members in hepatocytes from the same rats (A1, B1, C1, D1) were exposed to either the episodic male-like GH profile or the hormone vehicle for 6 days in culture. Results are presented and statistically analyzed as described in Fig. 4B2.

with the male-dependent profile suggesting irreversible sex-dependent imprinting (Thangavel \& Shapiro 2007).

Hepatocytes from the control treatment group exposed to the male-like GH profile responded with a 2to 3-fold increase in CIS, SOCS2 and SOCS1 expression levels (Fig. 6A2, B2 and C2). In contrast, the SOCS expression levels in adult hepatocytes from the MSG-alone treatment group were actually suppressed by the episodic GH profile, whereas cells from the combined MSG and $\mathrm{GH}$ neonatal treatment responded the same as controls to the masculine-like GH profile. As with other measured parameters, hepatocytes derived from GH alone-treated rats exhibited a muted response of CIS, SOCS2 and SOCS1 to the episodic GH profile. 


\section{GH imprinting is associated with permanent alterations in miRNA expression}

miRNAs are naturally occurring small, singlestranded non-coding RNA molecules involved in posttranscriptional control of gene expression. In animals, miRNAs imperfectly hybridize, through partial sequence homology, to the 3'-UTR of target mRNAs causing a block in translation and/or increased mRNA degradation (Bartel 2009). Prediction of miRNA target mRNA was carried out by our use of the algorithms miRanda (John et al. 2004) and TargetScan (Lewis et al. 2003). Four miRNAs were identified by in silico analyses with high predictability to interfere with the expression and/or action of CYP2C11 and/or GH. Three of the miRNAs (miR-29b-3p, miR-130a-3p and miR-381-3p) exhibited a dramatic $\geq 3$-fold overexpression in freshly isolated hepatocytes from GH-devoid MSG and MSG plus GH-treated rats
(Fig. 7A1, B1 and C1). As these 3 miRNAs have high predictability pairing scores for such relevant mRNAs as Cyp2c11, Socs2 and Igf1 (Fig. 7D) known to be regulated by the masculine $\mathrm{GH}$ profile, their overexpression is in agreement with the subnormal in vivo expression levels of CYP2C11 (Fig. 4B1), SOCS2 (Fig. 6B1) and IGF1 (Pampori \& Shapiro 1996) in episodic GH-ablated male rats. Furthermore, the 3 miRNAs were found to be completely unresponsive to the in vitro administration of the episodic $\mathrm{GH}$ profile in heptatocytes from all treatment groups neonatally exposed to $\mathrm{GH}$, i.e. controls, $\mathrm{GH}$ alone and MSG + GH (Fig. 7A2, B2 and C2). Only hepatocytes derived from the MSG-alone treatment responded to the episodic GH profile with a significant reduction in miR-29b-3p and miR130a-3p concentrations, now similar to all the other treatment groups indicating that neonatal GH imprints the 3 miRNAs to be unresponsive to episodic
A1

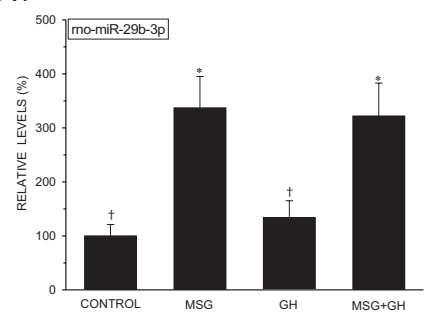

B1
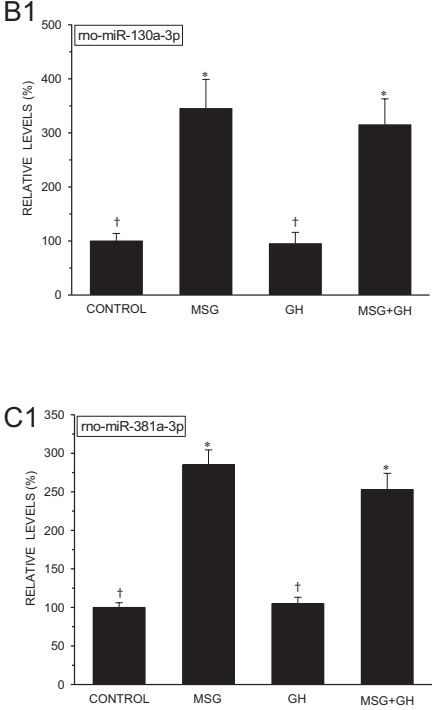

A2

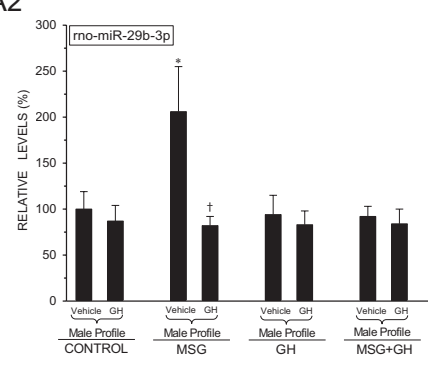

B2

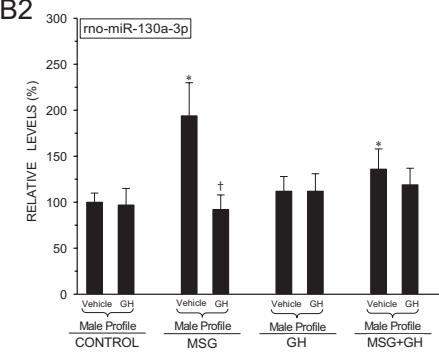

C2

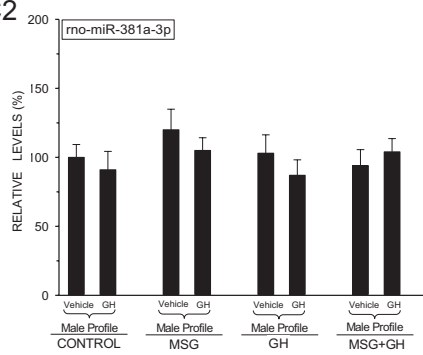

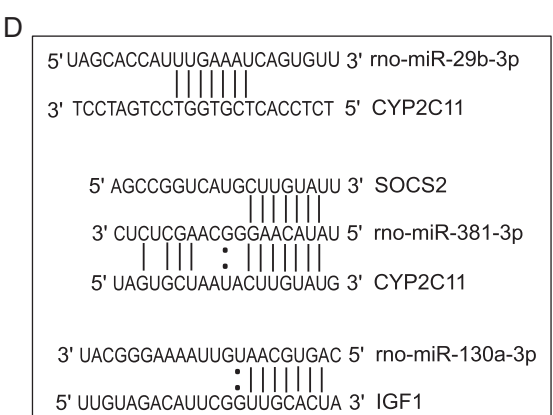

5' uUguagacauucgGUUGCACUA 3' IGF1

\section{Figure 7}

Adult expression of 3 rat-specific hepatic microRNAs (miR) neonatally imprinted by growth hormone (GH). (A1, B1, C1) Levels of 3 microRNAs (rno-miR29b-3p, rno-miR-381a-3p, rno-miR-130a-3p) were measured in freshly isolated preplated hepatocytes from adult male rats neonatally administered diluents (CONTROL), MSG, GH or MSG + GH as described and statistically analyzed in Fig. 1. (A2, B2, C2) Levels of the same hepatic microRNAs from the same rats $(A 1, B 1, C 1)$ were exposed to either the episodic male-like GH profile or the hormone vehicle for 6 days in culture. Results are presented and statistically analyzed as described in Fig. 4B2. (D) Sequence of the 3 microRNAs and their complementary sites in the 3'-UTR of CYP2C11, SOCS2 and IGF1.

http://joe.endocrinology-journals.org DOI: $10.1530 / J O E-16-0471$ (c) 2017 Society for Endocrinology Printed in Great Britain
Published by Bioscientifica Ltd 
GH regulation. (miRNA-141-3p, an in silico predicted blocker of STAT5b expression, was unaffected, statistically, by all the neonatal treatments (data not presented)).

\section{Discussion}

As an endocrine-responsive tissue appears to be imprinted/ programmed by the same hormone that regulates its adult function (Shapiro \& Goldman 1979, Csaba 2008), it seemed reasonable to assume that the expression of adult hepatic CYPs by GH is accordingly imprinted by GH. To investigate the specific requirement for perinatal $\mathrm{GH}$ in the imprinting process, we blocked postpartum GH secretion in newborn male rats using MSG, a selective disrupter of developing hypothalamic GH-releasing hormone nuclei (Bakke et al. 1978, Dada et al. 1984) while having no direct effect on liver differentiation (Kaufhold et al. 2002). In addition, some of the MSG-treated neonates simultaneously received replacement rat $\mathrm{GH}$ during the 'critical developmental period'. We also included two control groups: vehicle alone or $\mathrm{GH}$ alone. Plasma GH levels were normal in both control groups during the first two weeks of life, whereas neonatal MSG virtually eliminated circulating $\mathrm{GH}$ after the first injection, and as designed, concurrent $\mathrm{GH}$ replacement maintained generally normal postnatal levels of the hormone. However, as soon as exogenous GH was withdrawn, plasma concentrations of the hormone declined to undetectable as observed in the MSG alone group. As adults, males from both control groups secreted the typical masculine GH profile (Pampori et al. 1991b, Agrawal \& Shapiro 2000) characterized by episodic pulses of $\sim 1 \mathrm{~h}$ duration separated by interpulses of $2-3 \mathrm{~h}$ containing undetectable concentrations of the hormone. The one exception being that the pulse amplitudes were somewhat lower in the GH alone group. Since the GH-releasing hormone's hypothalamic nuclei were permanently destroyed in the neonates by MSG, both MSG groups, with and without concurrent neonatal GH replacement, secreted no measurable levels of GH as adults. Nevertheless, and in spite of the same complete lack of circulating $\mathrm{GH}$, the two MSG treatment groups expressed very different peripubertal and adult functions of what would normally be GH-dependent activities. That is, adults from the MSGalone treatment group were profoundly obese, exhibited a 3-fold longer hexobarbital-induced sleep time than controls and a concomitant near-complete deficiency in hexobarbital hydroxylase-dependent CYP2C11. In contrast and irrespective of the same absence of plasma
$\mathrm{GH}$, adult males neonatally treated with both MSG and GH exhibited only a late-onset mild obesity, a prolonged hexobarbital-induced sleep time that was still $\sim 30 \%$ shorter than that resulting from the MSG-alone treatment and CYP2C11 expression levels (mRNA and protein) representing $\sim 40 \%$ of controls. Evidently, even though there was no circulating GH after 12 days of age in the MSG + GH treatment group, the neonatal replacement $\mathrm{GH}$ alone appears to have programmed a permanent imprint that attenuated the abnormalities associated with congenital-like GH deficiency as characterized by the MSG alone treatment. The present findings identifying the effects of $\mathrm{GH}$ imprinting are analogous to those of the model developmental organizer/imprinter androgen. During the perinatal period when the brain is nonsexual and still differentiating, testicular androgens or one of their metabolites programme/imprint neural pathways required to fully express numerous masculine functions, such as gonadotropin secretory profiles, taste preferences and sexual behavior, when subsequently exposed to adult androgens (Feder 1981, Shapiro 1985a). Without hormonal imprinting, however, these masculine neural pathways are never established and/or are permanently refractory to androgens and their dependent masculine functions can never be expressed, regardless of adult hormone treatment. In this regard, it seemed more than reasonable to assume that androgens also masculinize (i.e., imprint) hepatic sex-dependent CYP isoforms, as neonatal orchidectomy had been reported to demasculinize drug metabolism (i.e., CYP isoforms). However, the permanence or irreversibility of the imprinting was either never tested by administering testosterone to neotnatal castrates as adults (Chung \& Chao 1980, Waxman et al. 1985) or, in fact, the defects produced by neonatal castration were found to be completely corrected when treated with the androgen as adults (Gustafsson \& Stenberg 1974, Jansson et al. 1985b, Dannan et al. 1986, Bandiera \& Dworschak 1992). (Androgens, including testosterone, have no direct effect on hepatic CYP expression, but rather can act at the hypothalamic-pituitary axis to regulate the sexually dimorphic secretion of GH, which, in turn, regulates sex-dependent CYPs (Kramer et al. 1979, Jansson et al. 1985a, Shapiro 1985b)). If the effects of neonatal orchidectomy on CYP expression can be completely corrected (i.e., reversible), by definition, and despite the researchers' conclusions, androgens do not imprint the sexually dimorphic expression of CYP isoforms. Moreover, our observation that neonatal MSG has no inhibitory effects on circulating testosterone levels during the first 15 days of life, inclusive of the

Published by Bioscientifica Ltd. 
critical period of hypothalamic-pituitary-hepatic axis differentiation (Das et al. 2014), is further support that perinatal androgens are not imprinters of CYP sexual dimorphisms. Rather, our results indicate that just as the brain of the adult not imprinted by androgens is permanently incapable of responding to androgens with a display of masculine behaviors, the liver of the adult male rat not imprinted by $\mathrm{GH}$ is permanently incapable of responding to episodic GH induction of the predominant CYP2C11 isoform. Consequently, hepatocytes derived from adult males not imprinted by neonatal GH (MSG only treatment) were completely unresponsive to episodic GH induction of CYP2C11 expression. In contrast, when the same neonatal treatment was supplemented with rat $\mathrm{GH}$, the adult hepatocytes were fully responsive to the episodic GH profile exhibiting a $\sim 2.5$-fold induction of CYP2C11 establishing perinatal $\mathrm{GH}$ as a requisite developmental organizer or imprinter.

What, then, are the defects in the GH-inductive response of CYP2C11 in adult rats neonatally treated with MSG? That is, why cannot the hepatocytes respond to GH? In this regard, GH signaling in liver by the episodic profile (in contrast to the continuous profile) is initiated by hormone binding to and the resulting activation of $\mathrm{GH}$ receptor (GHR) on the surface of the target cells. This allows for the recruitment and/or activation of two molecules of Janus kinase 2 (JAK2), which then cross-phosphorylate each other, as well as phosphorylating the receptor on key tyrosine residues. STAT5b, a latent transcription factor, binds to these phosphorylated receptor docking sites and is, in turn, phosphorylated and homodimerizes and translocates to the nucleus, where it binds to promoter sites initiating transcription of GH-regulated genes. An important negative regulatory mechanism of GH signaling, the SOCS family of inhibitory proteins, which, depending on the protein, inhibit GH signaling by inactivating JAK2, by competing with STAT5b for common tyrosinebinding sites on the intracellular tail of the GHR and/or by a proteasome-dependent mechanism that results in the degradation of the (GH-GHR-JAK2)•SOCS complex (Waxman \& Frank 2000, Landsman \& Waxman 2005, LeRoith \& Nissley 2005). At least 4 possible components in this signal transduction pathway were permanently altered by neonatal MSG. The response to episodic GH of hepatocytes from control rats (vehicle-treated more so than GH alone; the latter suggesting that too much of a hormone, just like too little, can have deleterious effects) increased cellular CIS, SOCS1 and SOCS2, as well as nuclear PSTAT5b, by 2 - to 3 -fold, whereas these transducers in hepatocytes from the MSG-treated rats were completely unresponsive to the masculine GH profile. Not only did developmental exposure to MSG permanently suppress STAT5b activation (i.e. phosphorylation) and nuclear translocation, but it also completely blocked the binding of the transcription factor to the Cyp2c11 promoter, explaining the inability of the masculine GH profile to induce CYP2C11 expression. In contrast, when MSG-treated pups were briefly injected with rat GH, induction levels of cellular CIS, SOCS1 and SOCS2 as well as nuclear translocation levels of pSTAT5b and its subsequent binding to the Cyp2c11 promoter were statistically the same as vehicle controls demonstrating the successful imprinting of the developing liver by neonatal $\mathrm{GH}$ alone. The possible involvement of miRNAs in the suppression of adult CYP2C11 by neonatal MSG was also examined. We used multiple in silico prediction tools and identified complimentary sequence homology sites in downstream GH-activated genes, including Cyp2c11 and Socs2. Three of the miRNAs, miR-29b, miR-130a and miR-381 were several fold overexpressed only in the adult GH-devoid, MSG-treated males in agreement with a report that miR-29b is suppressed by GH treatment (Cheung et al. 2009). Although it is not clear just what role, if any, the miRNAs play in GH imprinting, it is apparent from our findings that all three miRNAs are permanently refractory to GH regulation in only those animals neonatally imprinted by GH (i.e., vehicle alone, GH alone and $\mathrm{MSG}+\mathrm{GH}$ ). As miRNA studies are a nascent area of biological investigation, any conclusion that one of the purposes of GH imprinting is to permanently limit the inhibitory effects of miRNA on adult GH induction of CYP2C11 and other GH-dependent genes may seem reasonable (Banerjee et al. 2015), but remains speculative.

Clearly, then, neonatal exposure to MSG irreversibly suppresses GH activation of CYP2C11-dependent signal transduction at multiple steps. Whether the multiple defects in the signaling pathway are all independently induced or rather the result of an initial upstream defect (i.e. GHR) cascading dysfunctions down the signaling pathway is under investigation. In summary, our findings demonstrate for the first time that perinatal GH imprints a latently expressed programme, which is then subsequently responsive to $\mathrm{GH}$ during adulthood. The imprinting is obligatory and occurs during a critical developmental period, whereas the imprinted programme is only expressed in adulthood. As there is a myriad of metabolic functions in humans, including growth rates and lean body mass (e.g., note our Lee index findings); cardiovascular, bone, adipose and muscle physiology; protein, carbohydrate, lipid and electrolyte metabolism all regulated, at least in part, by GH, and like CYP expression, exhibit permanent

Published by Bioscientifica Ltd. 
sexual dimorphisms (Thangavel \& Shapiro 2007), it may be that these adult functions also require perinatal $\mathrm{GH}$ imprinting and could be vulnerable to pediatric drug therapy known to inadvertently disrupt GH secretion.

\section{Declaration of interest}

The authors declare that there is no conflict of interest that could be perceived as prejudicing the impartiality of the research reported.

\section{Funding}

This work was supported by the U.S. National Institutes of Health, Eunice Kennedy Shriver National Institute of Child Health and Human Development (grant HD-061285).

\section{Acknowledgements}

The authors thank Sarah Rauers for proofreading the manuscript.

\section{References}

Agrawal AK \& Shapiro BH 2000 Differential expression of genderdependent hepatic isoforms of cytochrome P-450 by pulse signals in the circulating masculine episodic growth hormone profile of the rat. Journal of Pharmacology and Experimental Therapeutics 292 228-237.

Agrawal AK, Pampori NA \& Shapiro BH 1995 Neonatal phenobarbitalinduced defects in age- and sex-specific growth hormone profiles regulating monooxygenases. American Journal of Physiology 268 E439-E445.

Bakke JL, Lawrence N, Bennett J, Robinson S \& Bowers CY 1978 Late endocrine effects of administering monosodium glutamate to neonatal rats. Neuroendocrinology 26 220-228. (doi:10.1159/000122829)

Bandiera S \& Dworschak C 1992 Effects of testosterone and estrogen on hepatic levels of cytochromes P450 2C7 and P450 2C11 in the rat. Archives of Biochemistry and Biophysics 296 286-295. (doi:10.1016/0003-9861(92)90574-G)

Banerjee S, Das RK \& Shapiro BH 2013 Growth hormone-independent suppression of growth hormone-dependent female isoforms of cytochrome $\mathrm{P} 450$ by the somatostatin analog ocreotide. European Journal of Pharmacology 715 256-261. (doi:10.1016/j. ejphar.2013.05.013)

Banerjee S, Das RK, Giffear KA \& Shapiro BH 2015 Permanent uncoupling of male-specific CYP2C11 transcription/translation by perinatal glutamate. Toxicology and Applied Pharmacology 284 79-91. (doi:10.1016/j.taap.2015.02.009)

Bartel D 2009 MicroRNAs: target recognition and regulatory functions. Cell 136 215-233. (doi:10.1016/j.cell.2009.01.002)

Brownborg HM, Klemcke HG, Borg KE \& Klindt J 1993 Prolactin and growth-hormone clearance in neonatal boars. Journal of Animal Science 71 2055-2060.

Cheung L, Gustavsson C, Norstedt G \& Tollet-Egnell P 2009 Sex-different and growth hormone-regulated expression of microRNA in rat liver. BMC Molecular Biology 10 13. (doi:10.1186/1471-2199-10-13)

Chung LWK \& Chao H 1980 Neonatal imprinting and hepatic cytochrome P-450. I. Comparison of testosterone hydroxylation in a reconstituted system between neonatally imprinted and nonimprinted rats. Molecular Pharmacology 18 543-549.

Csaba G 2008 Hormonal imprinting: phylogeny, ontogeny, diseases and possible role in present-day human evolution. Cell Biochemistry and Function 26 1-10. (doi:10.1002/cbf.1412)
Dada MO, Campbell GT \& Blake CA 1984 Effects of neonatal administration of monosodium glutamate on somatotrophs and growth hormone secretion in prepubertal male and female rats. Endocrinology 115 996-1003. (doi:10.1210/endo-115-3-996)

Dannan GA, Guengerich FB \& Waxman DJ 1986 Hormonal regulation of rat liver microsomal enzymes. Role of gonadal steroids in programming, maintenance, and suppression of $\Delta^{4}$-steroid $5 \alpha$-reductase, flavin-containing monooxygenase, and sex-specific cytochromes P-450. Journal of Biological Chemistry 261 10728-10735.

Das RK, Banerjee S \& Shapiro BH 2013a Noncanonical suppression of GH-dependent isoforms of cytochrome $\mathrm{P} 450$ by the somatostatin analog octreotide. Journal of Endocrinology 216 87-97. (doi:10.1530/ JOE-12-0255)

Das RK, Banerjee S \& Shapiro BH $2013 b$ Extensive sex- and/or hormonedependent expression of rat housekeeping genes. Endocrine Research 38 105-111. (doi:10.3109/07435800.2012.723294)

Das RK, Banerjee S \& Shapiro BH 2014 Irreversible perinatal imprinting of adult expression of the principal sex-dependent drug metabolizing enzyme CYP2C11. FASEB Journal 28 4111-4122. (doi:10.1096/fj.13248864)

Dhir RN, Dworakowski W, Thangavel C \& Shapiro BH 2006 Sexually dimorphic regulation of hepatic isoforms of human cytochrome p450 by growth hormone. Journal of Pharmacology and Experimental Therapeutics 316 87-94. (doi:10.1124/jpet.105.093773)

Dhir RN, Thangavel C \& Shapiro BH 2007 Attenuated expression of episodic growth hormone-induced CYP2C11 in female rats associated with suboptimal activation of the Jak2/Stat5B and other modulating signaling pathways. Drug Metabolism and Disposition 35 2102-2110. (doi:10.1124/dmd.107.017475)

Feder NH 1981 Perinatal hormones and their role in the development of sexually dimorphic behaviors. In Neuroendocrinology of Reproduction, Physiology and Behavior, pp 127-158. Ed NT Adler. New York, NY, USA: Plenum Press.

Goldman AS 1970 Animal models of inborn errors of steroidogenesis and steroid action. Hoppe-Seyler's Zeitschrift fur Physiologische Chemie $\mathbf{3 5 1}$ 426-436.

Gustafsson JA \& Stenberg A 1974 Masculinization of rat liver enzyme activities following hypophysectomy. Endocrinology 95 891-896. (doi:10.1210/endo-95-3-891)

Hannemann F, Bichet A, Ewen KM \& Bernhardt R 2007 Cytochrome P450 systems-biological variations of electron transport chains. Biochimica et Biophysica Acta 1770 330-344. (doi:10.1016/j.bbagen.2006.07.017)

Jansson JO, Eden S \& Isaksson O 1985a Sexual dimorphism in the control of growth hormone secretion. Endocrine Reviews 6 128-150. (doi:10.1210/edrv-6-2-128)

Jansson JO, Ekberg S, Isaksson O, Mode A \& Gustafsson JA 1985b Imprinting of growth hormone secretion, body growth, and hepatic steroid metabolism by neonatal testosterone. Endocrinology 117 1881-1889. (doi:10.1210/endo-117-5-1881)

John B, Enright AJ, Aravin A, Tuschl T, Sander C \& Marks DS 2004 Human microRNA targets. PLoS Biology 2 1862-1879.

Kacsoh B, Terry LC, Meyers JS, Crowley WR \& Grosvenor CE 1989 Maternal modulation of growth hormone secretion in the neonatal rat. I. Involvement of milk factors. Endocrinology 125 1326-1336. (doi:10.1210/endo-125-3-1326)

Karlsson H, Gustafsson JA \& Mode A 1999 Cis desensitizes GH induced Stat5 signaling in rat liver cells. Molecular and Cellular Endocrinology 154 37-43. (doi:10.1016/S0303-7207(99)00101-X)

Kaufhold A, Nigam PK, Dhir RN \& Shapiro BH 2002 Prevention of latently expressed CYP2C11, CYP3A2, and growth hormone defects in neonatally monosodium glutamate-treated male rats by the $\mathrm{N}$-methyl-d-aspartate receptor antagonist dizocilpine maleate. Journal of Pharmacology and Experimental Therapeutics 302 490-496. (doi:10.1124/jpet.102.034785)

Kramer RE, Greiner JW, Rumbaugh RC, Sweeney TD \& Colby HD 1979 Requirement of the pituitary gland for gonadal hormone effects

Published by Bioscientifica Ltd 
on hepatic drug metabolism in rats. Journal of Pharmacology and Experimental Therapeutics 208 19-23.

Landsman T \& Waxman DJ 2005 Role of the cytokine-induced SH2 domain-containing protein CIS in growth hormone receptor internalization. Journal of Biological Chemistry $28037471-37480$. (doi:10.1074/jbc.M504125200)

Legraverend C, Mode A, Wells T, Robinson I \& Gustafsson JA 1992 Hepatic steroid hydroxylating enzymes are controlled by the sexually dimorphic pattern of growth hormone secretion in normal and dwarf rats. FASEB Journal 6 711-718.

Leroith D \& Nissley P 2005 Knock your SOCS off! Journal of Clinical Investigation 115 233-236. (doi:10.1172/JCI24228)

Lewis BP, Shih IH, Jones-Rhoades MW, Bartel DP \& Burge CB 2003 Prediction of mammalian microRNA targets. Cell 115 787-798. (doi:10.1016/S0092-8674(03)01018-3)

Morgan ET, MacGeoch C \& Gustafsson JA 1985 Hormonal and developmental regulation of expression of the hepatic microsomal steroid 16 alpha-hydroxylase cytochrome P-450 apoprotein in the rat. Journal of Biological Chemistry 260 11895-11898.

Nebert DW \& Dieter MZ 2000 The evolution of drug metabolism. Pharmacology 61 124-135. (doi:10.1159/000028393)

Ohno S 1979 Major sex-determining genes. Berlin, Germany: SpringerVerlag.

Oliver C, Giraud P, Lissitzky JC, Cote J, Boudouresque F, Gillioz P \& Conte-Devolx B 1982 Influence of endogenous somatostatin on growth hormone and thyrotropin secretion in neonatal rats. Endocrinology 110 1018-1022. (doi:10.1210/endo-110-3-1018)

Olney JW 1969 Brain lesions, obesity and other disturbances in mice treated with monosodium glutamate. Science 164 719-721. (doi:10.1126/science.164.3880.719)

Pampori NA \& Shapiro BH 1996 Feminization of hepatic cytochrome P450s by nominal levels of growth hormone in the feminine plasma profile. Molecular Pharmacology 50 1148-1156.

Pampori NA \& Shapiro BH 1999 Gender differences in the responsiveness of the sex-dependent isoforms of hepatic P450 to the feminine plasma growth hormone profile. Endocrinology 140 1245-1254. (doi:10.1210/en.140.3.1245)

Pampori NA, Agrawal AK, Waxman DJ \& Shapiro BH 1991a Differential effects of neonatally administered glutamate on the ultradian pattern of circulating growth hormone regulating expression of sexdependent forms of cytochrome P450. Biochemical Pharmacology 41 1299-1309. (doi:10.1016/0006-2952(91)90101-A)

Pampori NA, Agrawal AK \& Shapiro BH $1991 b$ Renaturalizing the sexually dimorphic profiles of circulating growth hormone in hypophysectomized rats. Acta Endocrinology 124 283-289. (doi:10.1530/acta.0.1240283)

Ryan DE \& Levin W 1990 Purification and characterization of hepatic microsomal cytochrome P-450. Pharmacology and Therapeutics 45 153-239. (doi:10.1016/0163-7258(90)90029-2)

Shapiro BH 1985a A paradox in development: masculinization of the brain without androgen receptors. Progress in Clinical and Biological Research 171 151-173.

Shapiro BH $1985 b$ Pituitary-dependent masculinization of hepatic hexobarbital hydroxylase in Crl: CD-1(ICR)BR mice. Life Sciences 36 1169-1174. (doi:10.1016/0024-3205(85)90234-6)

Shapiro BH \& Goldman AS 1979 New thoughts on sexual differentiation of the brain. In Genetic Mechanisms of Sexual
Development, pp 221-252. Eds IH Porter \& HL Vallet. New York, NY, USA: Academic Press.

Shapiro BH, Levine DC \& Adler NT 1980 The testicular feminized rat: a naturally occurring model of androgen independent brain masculinization. Science 209 418-420. (doi:10.1126/science.7384816)

Shapiro BH, MacLeod JN, Pampori NA, Morrissey JJ, Lapenson DP \& Waxman, DJ 1989 Signalling elements in the ultradian rhythm of circulating growth hormone regulating expression of sex-dependent forms of hepatic cytochrome P450. Endocrinology 125 2935-2944. (doi:10.1210/endo-125-6-2935)

Shapiro BH, Agrawal AK \& Pampori NA 1995 Gender differences in drug metabolism regulated by growth hormone. International Journal of Biochemistry and Cellular Biology 27 9-20. (doi:10.1016/13572725(94)00056-5)

Thangavel C \& Shapiro BH 2007 A molecular basis for the sexually dimorphic response to growth hormone. Endocrinology 148 2894-2903. (doi:10.1210/en.2006-1333)

Thangavel C \& Shapiro BH 2008 Inherent sexually dimorphic expression of hepatic CYP2C12 correlated with repressed activation of growth hormone-regulated signal transduction in male rats. Drug Metabolism and Disposition 36 1884-1895. (doi:10.1124/dmd.108.021451)

Thangavel C, Garcia MC \& Shapiro BH 2004 Intrinsic sex differences determine expression of growth hormone-regulated female cytochrome P450s. Molecular and Cellular Endocrinology 220 31-39. (doi:10.1016/j.mce.2004.04.002)

Thangavel C, Dworakowski W \& Shapiro BH 2006 Inducibility of malespecific isoforms of cytochrome $\mathrm{p} 450$ by sex-dependent growth hormone profiles in hepatocyte cultures from male but not female rats. Drug Metabolism and Disposition 34 410-419.

Thangavel C, Boopathi E \& Shapiro BH 2011 Intrinsic sexually dimorphic expression of the principal human CYP3A4 correlated with suboptimal activation of GH/glucocorticoid-dependent transcriptional pathways in men. Endocrinology $1524813-4824$. (doi:10.1210/en.2011-1274)

Tokuyama K \& Himms-Hagen J 1986 Brown adipose tissue thermogenesis, torpor, and obesity of glutamate-treated mice. American Journal of Physiology 250 E409-E415.

Verma AS, Dhir RN \& Shapiro BH 2005 Inadequacy of the Janus kinase 2/ signal transducer and activator of transcription signal transduction pathway to mediate episodic growth hormone-dependent regulation of hepatic CYP2C11. Molecular Pharmacology 67 891-901. (doi:10.1124/mol.104.005454)

Waxman DJ \& Frank SJ 2000 Growth hormone action: signaling via a JAK-STAT-coupled receptor. In: Principles of Molecular Regulation, pp 55-83. Eds MP Conn \& AR Means. Totowa, NJ, USA: Humana Press.

Waxman DJ, Dannan GA \& Guengerich FB 1985 Regulation of rat hepatic cytochrome P-450: age dependent expression, hormonal imprinting, and xenobiotic inducibility of sex-specific isoenzymes. Biochemistry 24 4409-4417. (doi:10.1021/bi00337a023)

Waxman DJ, Pampori NA, Ram PA, Agrawal AK \& Shapiro BH 1991 Interpulse interval in circulating growth hormone patterns regulates sexually dimorphic expression of hepatic cytochrome P450. PNAS $\mathbf{8 8}$ 6868-6872. (doi:10.1073/pnas.88.15.6868)

Wehrenberg WB \& Giustina A 1992 Basic counterpoint: mechanisms and pathways of gonadal steroid modulation of growth hormone secretion. Endocrine Reviews 13 299-308. (doi:10.1210/edrv-13-2-299)

Received in final form 6 December 2016

Accepted 15 December 2016

Accepted Preprint published online 15 December 2016 http://joe.endocrinology-journals.org

DOI: 10.1530/JOE-16-0471
๑) 2017 Society for Endocrinology Printed in Great Britain
Published by Bioscientifica Ltd 